\title{
Feasibility and Effectiveness of Adding an Approach Avoidance Training With Game Elements to a Residential Childhood Obesity Treatment - A Pilot Study
}

\author{
Sandra Verbeken,' Wouter J. Boendermaker, ${ }^{2}$ Tom Loeys, ${ }^{3}$ Ellen Moens,' \\ Lien Goossens,' Julie Latomme ${ }^{4}$ and Caroline Braet' \\ I Department of Developmental, Personality and Social Psychology, Ghent University, Ghent, Belgium \\ ${ }^{2}$ Department of Developmental Psychology, University of Amsterdam, Amsterdam, The Netherlands \\ ${ }^{3}$ Department of Data Analysis, Ghent University, Ghent, Belgium \\ ${ }^{4}$ Department of Movement and Sports Sciences, Ghent University, Ghent, Belgium
}

\begin{abstract}
Childhood obesity treatment programs only result in moderate outcomes in the short term and do not reduce risk for future weight gain. Therefore, in the current study, Approach Avoidance Training (AAT) with motivational game elements will be added to an inpatient childhood obesity program with the aim of improving outcomes. Forty-one children (10-15 years) in the final months of an inpatient treatment program were randomised to either the AAT plus care-as-usual condition group or to a care-as-usual-only control group. During the 10 sessions, the children were trained to approach healthy food stimuli and to avoid unhealthy food stimuli. Treatment outcomes were child performances on tasks of AAT, implicit attitudes and attentional bias, self-report ratings on craving symptoms, and weight loss maintenance after leaving the clinic (12-week follow-up). Changes over time were not significantly different between conditions for the measures of automatic processes, craving, and weight loss maintenance. Possible accounts for the null findings, including sample size, influence of game elements, point of time in therapeutic process, limitations of the setting, and the control group are discussed. More research is needed before firm conclusions can be drawn about the clinical usefulness of CBM for weight control in childhood obesity.
\end{abstract}

Keywords: obesity, childhood, approach avoidance, attentional bias, craving, cognitive bias modification training

Childhood obesity is associated with considerable adverse physical, psychosocial and economical outcomes, both in the short term and the long term (Freedman, Khan, Dietz, Srinivasan, \& Berenson, 2001). Indeed, obesity is a serious medical disorder potentially leading to a number of comorbidities (e.g., cardiovascular diseases, diabetes, skeletal problems) and has a severe impact on the overall quality of life (Dixon, 2010). The most prominent consequence of childhood obesity seems to be the significantly increased risk of remaining obese into adulthood. In children aged between 10 and

Address for correspondence: Sandra Verbeken, Department of Developmental, Personality and Social Psychology, Ghent University, Ghent, Belgium.

E-mail: Sandra.Verbeken@UGent.be

Behaviour Change | Volume 35 | Number 2 | 2018 | pp. 91-107 | (C) The Author(s) 2018

doi 10.1017/bec.2018.11 
15 years, up to $80 \%$ of the obese children remained obese by age 25 (Whitaker, Wright, Pepe, Seidel, \& Dietz, 1997). Similar evidence emerged from a meta-analysis that included 25 longitudinal studies from around the world: all studies reported a moderate persistence of childhood overweight into adulthood (Singh, Mulder, Twisk, van Mechelen, \& Chinapaw, 2008). The finding that childhood obesity is not a transient developmental phenomenon is alarming given the association between adult obesity and several morbidities, and given the fact that extremely obese children may suffer cumulative effects of their 'pound-years' (Xanathakos \& Inge, 2007). Statistical and clinically meaningful weight loss can be achieved with behavioural lifestyle interventions (multidisciplinary obesity treatments; MOTs) focusing on diet, exercise and psychological techniques, including parental involvement (Braet, Tanghe, Decaluwé, Moens, \& Rosseel, 2004; Ho et al., 2013; Luttikhuis et al., 2009). However, those who successfully lost weight after MOT often regained weight when treatment was terminated (Braet, 2006; Moens, Braet, \& Van Winckel, 2010).

One explanation for the modest results of existing treatments is the decreased self-regulatory capacities of obese individuals (Smith, Hay, Campbell, \& Trollor, 2011). In several studies, compared to non-overweight children and despite similar IQ, age and social status, overweight children tended more often to disregard longterm goals and take the immediately available smaller reward (Graziano, Calkins, \& Keane, 2010; Nederkoorn, Braet, Van Eijs, Tanghe, \& Jansen, 2006; Seeyave et al., 2009; Verbeken, Braet, Claus, Nederkoorn, \& Oosterlaan, 2009; Verbeken, Braet, Bosmans, \& Goossens, 2014). In other words, they lack the skills to successfully resist temptation of the currently ever-present, calorie-rich food stimuli that automatically capture attention (Freijy, Mullan, \& Sharpe, 2014; Kemps, Tiggemann, \& Hollitt, 2014; Loeber et al., 2012; Mann \& Ward, 2004) and drive action tendencies to approach and eat palatable food (Brignell, Griffiths, Bradley, \& Mogg, 2009; Kemps \& Tiggemann, 2015; Kemps, Tiggemann, Martin, \& Elliott, 2013).

The assumption that in obese individuals overeating is triggered by exaggerated reactivity to stimuli associated with high-caloric food is supported by neuro-imaging research: compared to average weight controls, in obese individuals, a greater activation to high-caloric food images was found within a wide range of brain regions mediating motivational and attentional salience of food cues (Stoeckel et al., 2008; Yokum, Ng, \& Stice, 2011). Furthermore, increased brain activation to high-calorie food pictures in obese individuals predicted less weight loss during therapy and poorer weight-loss maintenance posttreatment (Murdaugh, Cox, Cook, \& Weller, 2012). Consequently, previous findings suggest that a high attentional bias for food cues is detrimental to weight control among obese individuals and that obesity treatment programs could benefit from approaches that target brain regions involved in attentional reactivity. As suggested by dual process models of behaviour regulation, obese children have both strong attentional bottom-up reactivity and a decreased top-down ability to inhibit the automatic impulses (Appelhans, 2009; Strack \& Deutch, 2004), leaving them at highest risk to become the plaything of environmental stimuli. Indeed, Guerrieri, Nederkoorn, and Jansen (2008) argue that focusing on inhibitory control alone will not be enough, without taking into account also attentional bottom-up processes. This is also in line with the view of Rollins, Dearing, and Epstein (2010) that bottom-up processes may be a more powerful independent predictor of food intake than the ability to inhibit.

Although MOTs already focus on improving impulse control by means of learning self-regulation skills (Braet et al., 2004; Duffy \& Spence, 1993), these 
vital skills seem hard to implement in daily life and are not very effective in the long term as children often relapse. It seems likely that, as long the supposed underlying core neurocognitive processes of poor impulse control are not modified, the acquired self-control skills remain of limited capacity. To reduce biased bottom-up processes, a number of cognitive bias modification (CBM) techniques were developed, aimed specifically at altering automatic, impulsive reactions to disorder-related cues (Koster, Fox, \& Macleod, 2009). In the domain of eating behaviour, several studies using adult samples suggest that CBM training may be effective for sustained weight loss (Kakoschke, Kemps, \& Tiggemann, 2014; Kemps, Tiggemann, \& Hollitt, 2015; Kemps, Tiggemann, Orr, \& Grear, 2014; Werthmann, Field, Roefs, Nederkoorn, \& Jansen, 2014). One study with obese children participating in an attention modification program (AMP) or control program (CC; Boutelle, Kuckertz, Carlson, \& Amir, 2014) showed that in a free access session, children in the CC condition significantly increased their caloric intake over time whereas children in the AMP group demonstrated decreased caloric intake, providing evidence for the effects of such a training on eating behaviour also in younger age groups. Further, a recent pilot study with obese children and adolescents (8-16 years) (Warschburger, Gmeiner, Morawietz, \& Rinck) showed that approachavoidance tendencies are trainable in this age group and that the training was well accepted.

As with any form of training, motivation to train plays an important role in reaching a positive treatment outcome, particularly when attempting to treat children and adolescents. Additionally, CBM tasks are often experienced as fairly boring, due to their repetitive nature (Beard \& Weisberg, 2012). The use of game elements to improve motivation to train may help with this problem (Gladwin, Figner, Crone, $\&$ Wiers, 2011). While the use of game elements in the field of CBM is still very limited (Boendermaker, Prins, \& Wiers, 2015), evidence from the field of executive function training showed promising evidence for the efficacy of a gamified EF-training as a weight stabilisation intervention in obese children (Verbeken, Braet, Goossens, \& van der Oord, 2013).

As such, the purpose of this pilot treatment study was to evaluate the feasibility and effectiveness of a novel CBM game training aimed at obese children above the care-as-usual of an intensive 10-month inpatient treatment program. Obese children were randomised to either the CBM training group or a control group provided with equal screen time but without food-related training elements (Tetris; www. TheTetrisGame.com). The CBM training aimed to reduce maladaptive cognitive biases by training both avoidance movements in response to high-caloric food pictures and approach movements in response to low-caloric food pictures. Primary outcome was weight loss maintenance at follow-up, three months after leaving the clinic. The training sessions were performed in the final months of the treatment program. In this way, after the end of the inpatient treatment program, confrontation with the daily food environment at home enabled us to study the long-term effects of a treatment in the natural environment, which is characterised by a high risk of relapse. Training prior to treatment could be useful in outpatient programs but not for inpatient programs, as during the 10-month program, no exposure to high caloric food is possible. We expected that the CBM training would reduce approach bias and craving for unhealthy high caloric food and that these changes would be highly needed when returning home and consequently be associated with better weight loss maintenance at follow-up. 


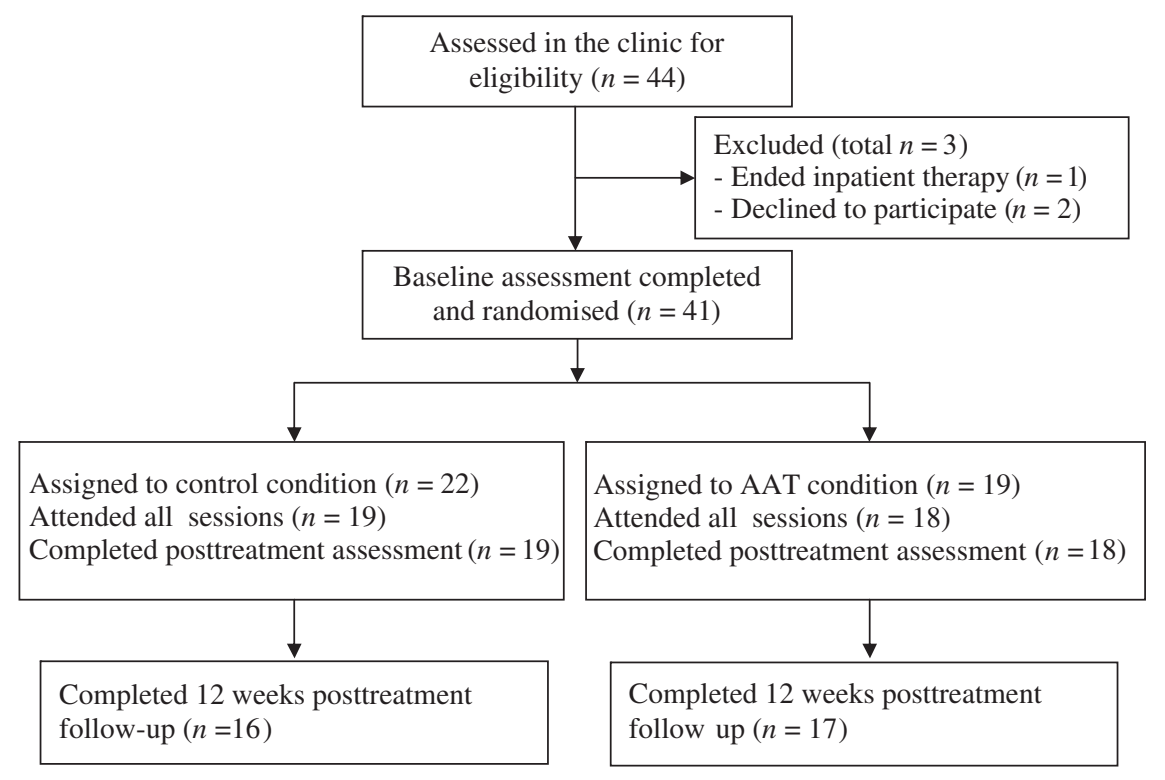

\section{FIGURE 1}

Flow of participants through the trial.

\section{Methods and Procedure}

\section{Participants}

The participants were obese children attending a 10-month inpatient obesity treatment program in a medical paediatric centre. Inclusion criteria for entering the treatment centre were: primary obesity, age between 10 and 15 years, IQ within the normal range as measured with the Raven Progressive Matrices (RPM; Raven, 1938), and absence of pervasive developmental disorders. Patients entered the clinic and started the obesity program in September. In February 2013, all 44 children entering the final phase of the obesity treatment program and their parents received an information letter about the research project in which they were invited to participate in month 8 of the treatment (see flow-chart in Figure 1). Forty children and their parents gave written consent (age $M=12.58$ years, $S D=1.43$; boys: $47.5 \%$ ). Three children and/or their parents declined to participate and one child dropped out of therapy before the study started.

\section{Measures}

94 Body weight. The body mass index (BMI, calculated as weight/height ${ }^{2}$ ) was determined for each child at pretraining, posttraining, and 12 weeks follow-up. Participants were defined as overweight if their BMI was at or above the 85th percentile, corresponding to a $z$ score of 0.97 , according to the Centers for Disease Control and Prevention (83rd CDC percentile). In order to make BMI comparisons between children and adolescents of different ages, an adjusted BMI was calculated as (actual $\mathrm{BMI} /$ percentile 50 of BMI for age and sex $) \times 100$, meaning a percentage over ideal BMI. However, since CDC $z$ scores are based on data of children who live in the 
United States, percentiles of the BMI for age and sex were based on normative Dutch data (Frederiks, van Buuren, Wit, \& Verloove-Vanhorick, 2000).

General Food Cravings Questionnaire - State (G-FCQ-S; Nijs, Franken, $\mathcal{E}$ Muris, 2007). The G-FCQ-S is a 15 -item, self-report scale measuring situational desire for food. The scale consists of five factors: (1) an intense desire to eat, (2) anticipation of relief from negative states and feelings as a result of eating, (3) craving as a physiological state, (4) obsessive preoccupation with food or lack of control over eating, and (5) anticipation of positive reinforcement that may result from eating. Participants were asked to rate how true each statement would be for them now, at this moment, using a 5-point scale ranging from 1 (totally not true) to 5 (totally true). A higher total score indicated that more craving was experienced in response to specific situations. The alpha coefficients in the present study for the pretest score was .84 and for the posttest score it was .96 .

General Food Cravings Questionnaire - Trait (G-FCQ-T; Nijs et al., 2007). The G-FCQ-T is a 21 -item, self-report scale measuring general desire for food and desire to eat. The scale consists of four factors: (1) preoccupation with food (i.e., obsessively thinking about food and eating), (2) loss of control (i.e., the tendency to demonstrate disinhibited eating behavior when exposed to food cues), (3) positive outcome expectancy (i.e., believing eating to be positively or negatively reinforcing), and (4) emotional craving (i.e., the tendency to crave food when experiencing negative emotions). Participants were asked to rate how true each statement would be for them now, at this moment, using a 6-point scale ranging from 1 (totally not true) to 6 (totally true). A higher total score indicated that more craving was experienced across times and situations. The alpha coefficients in the present study for the pretest score was.94 and for the posttest score it was .92 .

Approach/avoidance task. The approach avoidance task used in this study was an adaptation of Wiers, Rinck, Kordts, Houben, and Strack (2010), using a PC keyboard (the up and down arrow) instead of a joystick for responses. This measurement version used a set of 10 unique pictures of healthy food (e.g., a carrot) and 10 unique pictures of unhealthy food (e.g., chips), as well as four filler pictures of neutral stimuli (e.g., office supplies) for 16 practice trials (based on Pronk et al., 2015). The picture stimuli themselves were matched on various visual aspects; for example, white background, active (with person eating) or passive (food only). The pictures were all tilted slightly (3 degrees) to the left or to the right. Participants were only instructed to respond to the tilt of the pictures with an approach movement (arrow down key on the keyboard) to all pictures that were tilted 3 degrees to the left and to respond with an avoidance movement (arrow-up key) to pictures tilted 3 degrees to the right. This tilt-response contingency $($ left $=$ pull and right $=$ push, versus left $=$ push and right $=$ pull $)$ was counterbalanced over participants. To generate a sensation of approach or avoidance, making the approach movement increased the size of the picture, and pushing the picture away decreased its size. The instruction was to respond only to the tilt of the picture as quickly and accurately as possible, and to keep the key pressed down until the maximum or minimum zoom was reached (500 ms). As such, the required response was unrelated to the food depicted. The measurement phase consisted of 80 trials, where there was a 50/50 matching between tilt and content, that is, 50\% of the unhealthy pictures was tilted to the left and $50 \%$ to the right, and the same was true for the unhealthy pictures. Approach bias scores towards unhealthy food were 
calculated by subtracting the mean reaction times for unhealthy food-related pull trials from unhealthy food-related push trials. Similarly, bias scores toward healthy food were calculated by subtracting the mean reaction times for healthy food-related pull trials from healthy food-related push trials.

Implicit associations task. To see if the training effect on the approach/avoidance bias might translate to other cognitive processes, a single category implicit association task (SC-IAT) (Karpinski \& Steinman, 2006) was used to measure the children's implicit pleasant and unpleasant associations with unhealthy food. The SC-IAT is a shortened version of the implicit association task (IAT) originally developed by Greenwald, McGhee, and Schwartz (1998), which uses only one target category and is shortened to three blocks of trials. In the first block, 20 trials were used to practise with typical pleasant and unpleasant exemplar words, such as 'friendly' and 'angry', with the pleasant ('fun') or unpleasant ('annoying') labels respectively. The participants were instructed to categorise the words, which were presented in the centre of the screen, with either one of the two labels, presented on the left and right top of the screen. They did so by pressing the left or right arrow key on the keyboard respectively as quickly and accurately as possible. In the second block, a third category label 'food' was added, which only had unhealthy food exemplars (as there was no healthy food category, the category label was kept neutral) on either side of the screen, resulting in a response matching between 'fun' and 'food' on one side versus 'annoying' on the other, or vice versa, depending on the counterbalancing. In the final block, the 'food' label switched sides to match response key with the other label. The bias was calculated through the standardised difference in latencies (D score) between the second and third block, following the guidelines specified by Greenwald, Nosek, and Banaji (2003).

Attentional bias task. To see whether the training effect on the approach/avoidance bias might translate to other cognitive processes, a visual probe task (VPT) based on the task used by Schoenmakers et al. (2010) was used to measure the attentional bias for unhealthy food. During the VPT, two visually similar pictures, one of healthy and one of unhealthy food (based on Pronk et al., 2015) were shown simultaneously on the screen, positioned on the left and right side of the screen for $500 \mathrm{~ms}$. When the pictures disappeared, a small arrow was shown at the centre of the position where one of the pictures used to be. The arrow could point upwards or downwards, and the participant was instructed to respond to the arrow's direction as quickly and as accurately as possible by pressing the corresponding up or down arrow key on the keyboard. The placement of the healthy and unhealthy food picture was evenly distributed between the left and right position, and the arrow would appear in the unhealthy spot as often as in the healthy spot. The resulting mean reaction times on unhealthy food trials were subtracted from those on healthy food trials to calculate the attentional bias toward unhealthy food.

\section{Description of Interventions}

Inpatient treatment as usual. The inpatient treatment consisted of a 10-month, nondiet healthy lifestyle program. The aim was to achieve a healthy body weight through teaching the children to make healthy food choices at fixed times during the day, and to provide daily physical activities. Cognitive behavioural therapy (CBT) principals were integrated as part of the program. The program (description and evaluation 
in Braet et al., 2003) consists of three phases of approximately three months each: introduction phase, maintenance phase, and termination phase. A follow-up study showed that treated children lost a significant amount of overweight (with a mean loss of $50 \%$ ) over the 10-month period, whereas their non-treated case controls continued to gain weight (Braet, Tanghe, De Bode, Franckx, \& Van Winckel, 2003). In the last phase, when children were prepared for 'returning home' (termination phase), a mean of $10 \%$ additional weight loss was achieved, and at discharge, overweight was reduced to $20-30 \%$. However, follow-up data showed that after leaving the clinic, children regained some of their weight, and at the 14-month follow-up, the children had regained about $20 \%$ weight, resulting in about $44.1 \%$ of overweight (Braet et al., 2004). At the 6-year follow-up, overweight children returned to 53\% (Goossens, Braet, Verbeken, Decaluwe, \& Bosmans, 2011).

Approach Avoidance Training. An AAT game was developed, based on the approach/avoidance task, and was used to retrain the children's approach tendencies away from unhealthy food in 10 training sessions. Each training session took approximately 30 minutes and consisted of a training block of 12 minutes, followed by 3 minutes of 'game time break' (GTB), then another training block of 12 minutes and a final 3 minutes GTB. During these GTBs, the children were allowed to do bonus training trials to collect more points or to use their points in an online game. The session ended with an additional bonus block of 3 minutes maximum when the children were allowed to train for extra points, which could only be spent in the next session. Including bonus trials, the participants trained on 292 trials on average $(10 \%$ of which were filler trials) per session. Each correct response (i.e., matching the tilt with the correct response button) resulted in points, with a bonus for speed - for example, 1 point for a correct reaction time above 1,200 ms until 5 points for a correct reaction time below 300 ms. The daily achievement goal (in terms of points) was fixed, and equal for all children, only becoming a little higher each session.

The AAT used a 100/0 matching between tilt and content, that is, all unhealthy pictures were tilted to match the push (avoid) instruction, and all the healthy pictures were tilted to match the pull (approach) instruction. A set of 8 unique pictures of healthy food and 8 unique pictures of unhealthy food was used during each session, meaning each picture was repeated on average 18 times per session. At the start of each training session, the child was given a daily goal to gain a certain number of points, which upon achievement would earn them bonus points. The AAT was embedded inside an online game that allowed the children to use points earned through training to build a virtual city of little houses, trees, roads, and so on. A social element was added by letting the children view the cities of other participants, which they were also allowed to rate with a 'thumbs up'.

Treatment acceptability. After each session the child completed a diary assessing the acceptability of the computer task (training or Tetris). In particular, children were asked how much they liked the computer task on a 5-point Likert scale (from very nice to very boring) and how difficult they found the computer task on a 5-point Likert scale (from very difficult to very easy).

Tetris. The computer game Tetris was downloaded from www. TheTetrisGame.com and served as the control condition. This was done to allow us to keep two variables (screen time, game element) constant in both conditions. 


\section{TABLE 1}

Differences in Demographic Characteristics Between Children in the AAT and Control Conditions

\begin{tabular}{|c|c|c|c|}
\hline & $\begin{array}{l}\text { AAT } \\
(n=21) \\
\text { Mean }(S D)\end{array}$ & $\begin{array}{l}\text { Control } \\
(n=19) \\
\text { Mean }(S D)\end{array}$ & $F / \chi^{2}$ \\
\hline Age & $12.38(1.63)$ & $12.79(1.18)$ & .81 \\
\hline Sex (girls/boys) & $12 / 9$ & 9/10 & .38 \\
\hline Pretraining adjBMI & $145.77(22.30)$ & $136.69(16.07)$ & 2.14 \\
\hline
\end{tabular}

Note: $\operatorname{adjBMI}=$ adjusted body mass index.

\section{Procedure}

At the start of the 'returning home phase' after 6 months of inpatient treatment (April 2013) participants $(N=40)$ were randomly assigned to either a Tetris-control condition $(n=19)$ or to an active AAT condition (AAT, $n=21)$. Randomisation (using random number generator by a person blind to the study) was stratified on sex and age. After randomisation, the 10 computer sessions were spaced out over 4 weeks. The training took place on weekdays with approximately one non-training day in between, dependent on the therapeutic and school program of the children. Children were invited to a classroom and provided with a computer. It was ensured that this computer was placed at a location in the clinic with limited distractions.

Before the beginning of the AAT, the pretest was conducted (April 2013). The children were assessed in the clinic with the G-FCQ-T, G-FCQ-S, the Raven, the Visual Probe Task, the Approach Avoidance Task, and the Implicit Association Task. The assessment phase was spread over two days and took approximately 1.5 hours. One week after the training, participants in both conditions received the same posttest measures (June 2013), BMI was determined, and one week later the children left the clinic. A follow-up was 3 months after the posttest (September 2013), when children returned to the clinic for BMI determination (see flow chart in Figure 1). The assessors for the posttest and follow-up measures were blind to treatment condition. The Ethics Committee of the Ghent University approved the study. Demographic characteristics from children in both conditions are presented in Table 1.

\section{Data Analyses}

The primary outcome variable was adjusted BMI at 3 months' follow-up. Intermediate variables were changes in action tendencies (as registered on the AAT), changes in attentional bias (as registered on the VPT), changes in children's implicit pleasant and unpleasant associations of unhealthy food (as registered on the SC-IAT), and changes in craving (as measured with G-FCQ-S and G-FCQ-T).

ANOVAs and chi-square analyses were used for testing differences at baseline on outcome measures between participants of both treatment conditions. There were no significant differences between the two conditions on any of the variables. Outlier analysis showed no outliers on any of the dependent variables. During the training three children dropped out (two in the AAT group, one in the control group).

The change from baseline in action tendencies, attentional bias, implicit pleasant and unpleasant associations with unhealthy food and craving were analysed using 
TABLE 2

Pretraining Scores of the AAT Condition, Control Condition and Total Group

\begin{tabular}{|c|c|c|c|c|c|c|}
\hline & \multicolumn{2}{|c|}{ AAT $(n=19)$} & \multicolumn{2}{|c|}{ Control $(n=18)$} & \multicolumn{2}{|c|}{ Total $(N=37)$} \\
\hline & Mean & $(S D)$ & Mean & $(S D)$ & Mean & $(S D)$ \\
\hline AB-unh & -10.14 & $(43.06)$ & -17.78 & (37.74) & $-13.74 \dagger$ & (40.05) \\
\hline AATbias-unh & -43.78 & (102.55) & -33.26 & (116.55) & $-38.67 *$ & (108.07) \\
\hline AATbias-h & -39.78 & (104.71) & -39.59 & (96.22) & $-39.69 *$ & (99.19) \\
\hline IATbias & 0.02 & $(0.56)$ & 0.05 & $(0.45)$ & 0.03 & $(0.50)$ \\
\hline craving $T$ & 10.89 & $(3.60)$ & 8.84 & (3.51) & 9.84 & (3.65) \\
\hline cravingS & 14.20 & $(2.57)$ & 12.78 & (3.55) & 13.49 & (3.14) \\
\hline
\end{tabular}

Note: $A B-u n h=$ attentional bias score towards unhealthy food; AATbias-unh = approach bias score for unhealthy food; AATbias- $h=$ approach bias score for healthy food; IATbias = implicit association bias score; craving $\mathrm{T}=$ total score on the General Food Cravings Questionnaire - Trait; cravingS = total score on the General Food Cravings Questionnaire - State.

${ }^{*} p<.05, \dagger p<.10$

linear regression models with group as factor, adjusted for age and sex. The change in the adjusted BMI over time (pretraining, posttraining, follow-up) was assessed using linear mixed models with fixed effects for group, time as factor and group-by-time interaction, age and sex, and a correlated random intercept and random slope for time.

Finally, paired $t$ tests were used to analyse pre-post changes in bias and craving scores per condition.

\section{Results}

\section{Evaluation of AAT on Measures of Automatic Processes}

Pretraining scores of participants across conditions are shown in Table 2 . There were no significant differences in baseline values between both conditions. Overall, at baseline, the results showed a significant approach bias for unhealthy and healthy food (both $p<.05$ ), and a marginally significant attentional bias for unhealthy food $(p<.10)$.

Table 3 shows the mean change between scores on pretraining and posttraining for the bias scores, craving scores, and adjusted BMI in both conditions. The linear regression models revealed no significant differences between both conditions on any of the outcomes (all ps > .30).

In the AAT condition, paired $t$ tests showed from pre- to posttraining a significant reduction in approach bias toward unhealthy food cues $(p=.022)$ and toward healthy food cues $(p=.032)$, and a significant reduction in cravings $(p=.022)$. In the control group, no significant change over time was found for the different variables. The mean pre- and posttraining, bias and craving scores per condition are presented in Figure 2.

Weight change was measured comparing change on adjusted BMI between pretraining and posttraining follow-up. Figure 3 shows the change of the adjusted BMI over time. There were no significant differences between both conditions at any time point (all ps > .13). 
TABLE 3

Change Scores (Pre-Post Training) for the Different Outcome Measures

\begin{tabular}{lrrrrr}
\hline & \multicolumn{2}{c}{ AAT $(n=19)$} & & \multicolumn{2}{c}{ Control $(n=18)$} \\
\cline { 2 - 3 } \cline { 5 - 6 } & Mean & $(S D)$ & & Mean & $(S D)$ \\
\hline AB-unh & 5.94 & $(46.61)$ & & 16.31 & $(56.58)$ \\
AATbias-unh & 73.61 & $(123.97)$ & & 47.38 & $(146.15)$ \\
AATbias-h & 47.06 & $(85.26)$ & & 69.82 & $(152.72)$ \\
IATbias & -0.04 & $(0.58)$ & & 0.03 & $(0.51)$ \\
cravingT & -1.16 & $(3.46)$ & & -0.02 & $(2.64)$ \\
cravingS & -2.88 & $(4.15)$ & & -1.51 & $(3.79)$ \\
adjBMI & -7.37 & $(4.19)$ & & -8.35 & $(4.29)$ \\
\hline
\end{tabular}

Note: $A B-u n h=$ attentional bias score towards unhealthy food; AATbias-unh = approach bias score for unhealthy food; AATbias- $\mathrm{h}=$ approach bias score for healthy food; IATbias = implicit association bias score; craving $T=$ total score on the General Food Cravings Questionnaire - Trait; cravingS = total score on the General Food Cravings Questionnaire - State; adjBMI: adjusted BMI.

\section{Treatment Acceptability}

From the 19 children in the training condition, 10 completed the diaries. Among these children, 20\% experienced the training as boring, 50\% as neutral, and 30\% as nice to very nice (mean $=2.80, S D=.92$ ). Further, $10 \%$ experienced the training as very difficult, $50 \%$ as neutral, and $40 \%$ as easy to very easy (mean $=3.30, S D=1.06$ ).

\section{Discussion}

The current pilot study was conducted to evaluate the acceptability and effectiveness of adding an AAT with game elements to a 10-month inpatient treatment program for obese children. It was hypothesised that a retraining of automatic approach tendencies toward food cues embedded in a traditional care-as-usual model may help to weaken the children's automatic reactions to unhealthy food-related cues and help maintain initial weight loss.

For the main clinical outcome measure, no significant effects were found: the weight change between pretraining, posttraining and follow-up was the same in both conditions. For these obese children, a retraining of approach-avoidance tendencies toward healthy/unhealthy food cues did not seem to affect their weight-control capacity. This finding is in contrast with literature in adults and children, where CBM training results in decreased caloric intake and sustained weight loss (Boutelle et al., 2014; Kakoschke et al., 2014; Kemps \& Tiggemann, 2015; Kemps et al., 2014; Werthmann et al., 2014). The results also showed no significant group differences in approach and attentional bias change to food images over time, nor in the implicit associations and craving toward food from pre- to posttraining. Finally, results showed that overall, the training sessions had reasonable acceptability ratings from the children.

There may be several factors contributing to these findings. First, all children had lost significant weight during the inpatient treatment, and significant changes in their neuronal response to food-related cues might have been already established before the training intervention started. A decrease in activity in regions mediating motivational and attentional salience of food cues has previously been shown in people with a 

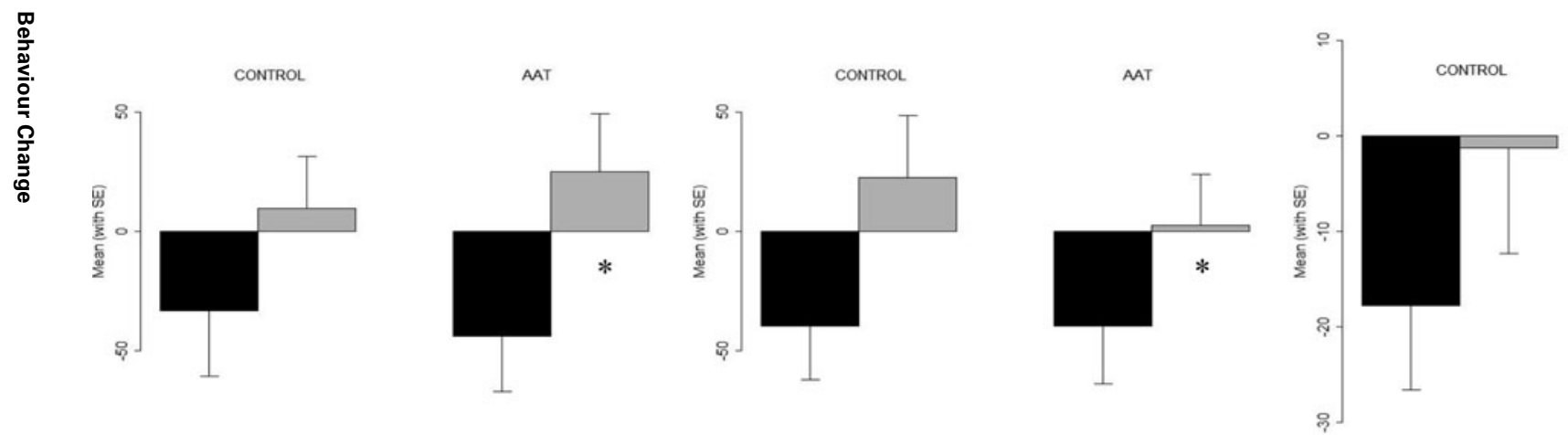

AAT

AAT Bias-unh Pre (black) and Post (grey)

AAT Bias.h Pre (black) and Post (grey)
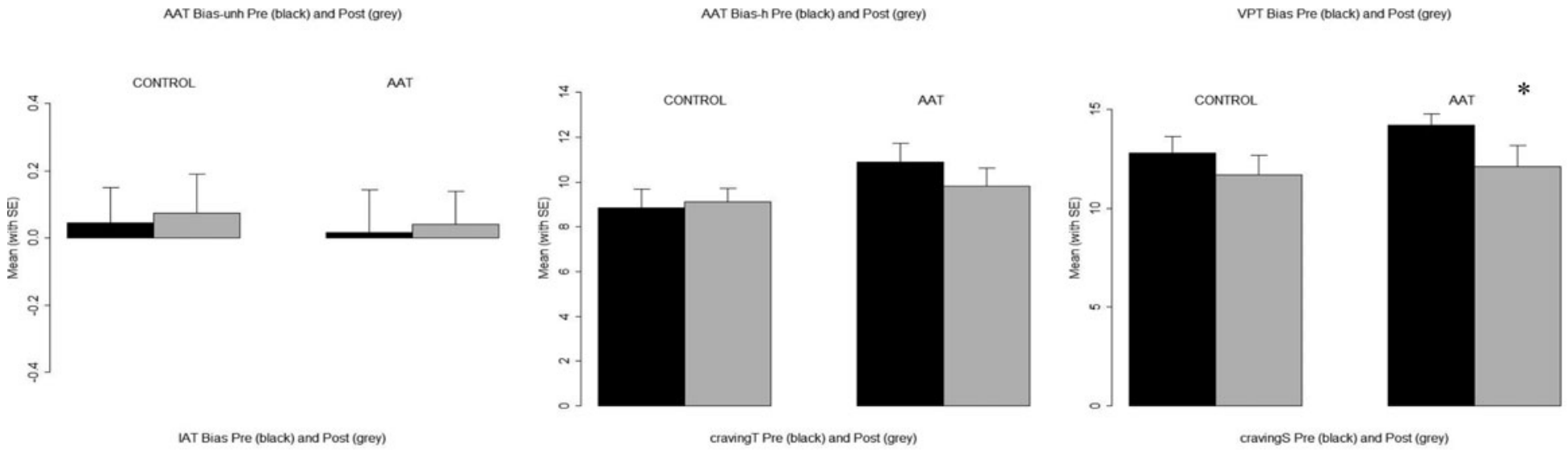

LAT Bias Pre (black) and Post (grey)

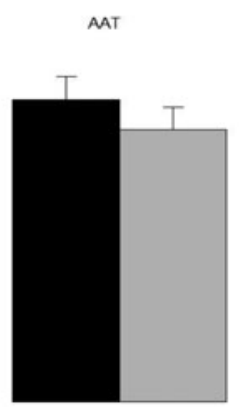

cravingS Pre (black) and Post (grey)

FIGURE 2

Graph of the mean approach bias scores, attention bias scores, IAT bias scores and craving scores (with standard errors) per condition (control/AAT). 


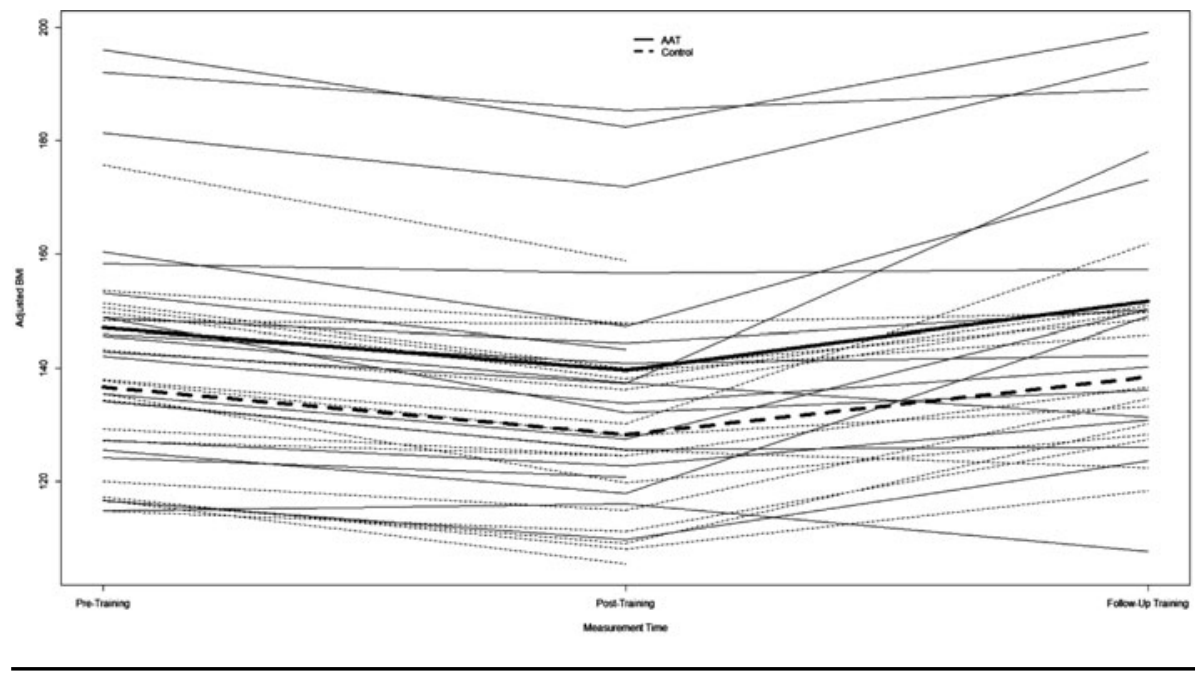

\section{FIGURE 3}

Evolution of the adjusted BMI over time for the AAT condition and control condition. Thick lines represent group means and fine lines represent individual scores.

weight-reduced state (Rosenbaum, Sy, Pavlovich, Leibel, \& Hirsch, 2008). This neuronal effect of weight reduction may have attenuated effective group differences due to the AAT. Furthermore, neuro-imaging research has shown decreased brain activation to high-calorie food cues in individuals following a cognitive-behavioural weight-loss treatment (Murdaugh, Cox, Cook, \& Weller, 2012). However, in the current study, the scores at pretraining, which is after 8 months of inpatient cognitive-behavioural weight-loss treatment and considerable weight loss, still reflect an approach bias for food present in these obese children.

Alternatively, attentional bias, approach tendencies and craving may be low overall due to the absence of tempting food cues within the treatment facility, making it less likely to capture training effects. Addiction literature has shown that attentional bias may peak during high-risk situations for relapse (Field, Marhe, \& Franken, 2014). Therefore, since the current food environment entails a continual 'high-risk' situation for obese children, future studies in ambulatory settings may be more adequate to evaluate the impact of the CBM training on approach and attentional bias and craving strength. Further, weight maintenance studies argue that in obesity treatment, initial weight response strongly predicts long-term outcome, and therefore it is recommended to facilitate in therapy early behavioural change (Goldschmidt et al., 2011; Jelalian et al., 2011). So, this means that implementing the training in the first weeks of treatment may be more effective, possibly with extra sessions as a maintenance training delivered via handheld devices such as smart phones that can be used when tempting cues are present or when craving is high.

Further, Boendermaker et al. (2015) put forward that the lack of game elements during the postmeasurement after a training game may demotivate participants to perform well and in this way possible training effects on the bias measures may also be indiscernible. In future research, game elements may be added to the measurement versions of the assessment tasks as well. Additionally, making it possible that training 
points could be collected across the entire task (from pre- to posttraining) may be also a way to avoid a decrease in motivation toward the end of the training.

However, it is also possible that the control condition was an active training. Recent research proved that playing Tetris may reduce craving strength and attentional bias (Skorka-Brown, Andrade, \& May, 2014). This research was built on the elaborated intrusion theory (Kavanagh, Andrade, \& May, 2005), implying that craving arises from visual images stored in the visuospatial sketchpad of working memory. The rapid rotations and movements of geometric shapes required during playing of Tetris loads heavily on the visuospatial working memory and competes with the encoding of visual 'craving-images' (Kavanagh et al., 2005). Van Dillen and Andrade (2016) found that playing Tetris was associated with reduced craving and attentional bias to food pictures and a reduced choice of high calorie food. Therefore, in future research, new control conditions must be selected (e.g., a version of the approach-bias retraining with $50 \%$ of unhealthy food pictures to be pushed).

Attentional bias modification is a retraining paradigm with promising results in addiction (Schoenmakers et al., 2010). In the current study, although between-condition changes were not significant, analyses per condition showed that our 10 sessions of AAT resulted in a significant reduction in approach tendencies toward unhealthy and healthy food pictures. This finding is in line with previous research showing that during a six-session AAT, obese children successfully learned to reduce their automatic approach tendencies toward snack food pictures, but otherwise the study of Warschburger and colleagues (2017) showed an increase in approach tendencies toward vegetable items. Further, the current training resulted also in a significant reduction in craving but failed to reduce the attentional bias toward unhealthy food pictures. Since attentional bias may be the more basic eliciting factor in the theoretical models (Franken, 2003; Yokum et al., 2011) and was shown in obesity research to be related to a greater responsiveness to food cues (Nijs et al., 2007), adding an attentional bias modification training may improve the effectiveness in reducing craving and enhancing weight-control.

Finally, the dual process model of obesity (Appelhans, 2009) suggests that executive functioning interacts with the automatic processes to resist temptation and regulate eating behaviour. It was shown that obese children and adolescents have decreased behavioural self-control capacities compared to normal weight peers: they lack the skills of resisting temptation (Grazaino et al., 2010; Nederkoorn et al., 2006; Seeyave et al., 2009; Verbeken et al., 2009; Verbeken et al., 2014). Basic research demonstrated that this can be explained by poor executive functions (EFs) needed to overrule automatic behaviours. EFs are neuropsychological processes that activate and regulate goal-directed behaviour and responses to the environment. It was also shown that EFs have an influence on obesity treatment, with impulsive individuals having more difficulties losing weight (Nederkoorn et al., 2007; Kulendran et al., 2014; Weygandt et al., 2013), maintaining weight loss (Weygandt et al., 2015), and leaving the treatment more often (Bjorvell \& Edman, 1989). Therefore, future research may need to include training of both bottom-up and top-down processes (embedded in MOT) to be effective for the sustained behaviour change needed to maintain initial weight loss and improve health outcomes.

A limitation of this study was certainly the rather low power. Replication in larger samples is required before firm conclusions about the effectiveness of AAT for obese children can be drawn. Although the current results prompt the tentative conclusion that AAT means no added value on top of the care-as-usual in childhood 
obesity treatment, it is possible that it is effective and helpful in a waitlist group of obese children. Further, adequately powered studies may include moderators in effect analyses. It is possible that AAT is more effective for children with higher BMI, higher craving scores, or stronger bias for unhealthy food at baseline.

To conclude, although significant effects were absent in the current pilot study, the fact that self-regulation is crucial for long-term successful weight control in children (Appelhans, 2009) and that this ability is undermined by strong automatic processes in obese children (Murdaugh et al., 2012; Yokum et al., 2011) makes further research on the added value of treatments to attenuate or retrain automatic processes in obese children essential. Future large-scale RCTs are needed, taking into account the lessons learned concerning the inclusion of motivational elements, the point of time in the therapeutic process, and the observed limitations of the setting and control group, before firm conclusions can be drawn about the clinical usefulness of CBM for weight control in childhood obesity. Further, also moderator analyses are needed.

\section{References}

Appelhans, B.M. (2009). Neurobehavioral inhibition of reward-driven feeding: Implications for dieting and obesity. Obesity, 17, 640-647.

Beard, C., \& Weisberg, R.B. (2012). Socially anxious primary care patients' attitudes toward cognitive bias modification (CBM): A qualitative study. Behavioural and Cognitive Psychotherapy, 40, 618633.

Bjorvell, H., \& Edman, G. (1989). Characteristics of drop-outs from a long-term behavioral treatment program for obesity. International Journal of Eating Disorders, 8, 363-368.

Boendermaker, W.J., Prins, P.J.M., \& Wiers, R.W. (2015). Cognitive bias modification for adolescents with substance use problems - Can serious games help? Journal of Behavior Therapy and Experimental Psychiatry, 9, 13-20. doi:10.1016/j.jbtep.2015.03.008.

Boutelle, K.N., Kuckertz, J.M., Carlson, J, . \& Amir, N. (2014). A pilot study evaluating a one-session attention modification training to decrease overeating in obese children. Appetite, 76, 180-185.

Braet, C. (2006). Patient characteristics as predictors of weight loss after an obesity treatment for children, Obesity, 14, 148-155.

Braet, C., Tanghe, A., De Bode, F., Franckx, H., \& Van Winckel, M. (2003). Inpatient treatment of obese children: A multicomponent programme without stringent calorie restriction. European Journal of Pediatrics, 162, 391-396. doi:10.1007/s00431-003-1155-5

Braet, C., Tanghe, A., Decaluwé, V., Moens, E., \& Rosseel, Y. (2004). Inpatient treatment for children with obesity: Weight loss, psychosocial well being, and eating behavior. Journal of Pediatric Psychology, 29, 519-529, doi:10.1093/jpepsy/jsh054

Brignell, C., Griffiths, T., Bradley, B.P., \& Mogg, K. (2009). Attentional and approach biases for pictorial food cues. Influence of external eating. Appetite, 52, 299-306.

Dixon, J.B. (2010). The effect of obesity on health outcomes. Molecular and Cellular Endocrinology, $316,104-108$.

Duffy, G., \& Spence, S.H. (1993). The effectiveness of cognitive self-management as an adjunct to a behavioural intervention for childhood obesity: A research note. Journal of Child Psychology and Psychiatry, 34, 1043-1050, doi: 10.1111/j.1469-7610.1993.tb01107.x

Field, M., Marhe, R., \& Franken, I.H.A (2014). The clinical relevance of attentional bias in substance use disorders. CNS Spectrums, 19, 225-230.

Franken, I.H.A. (2003). Drug craving and addiction: Integrating psychological and neuropsychopharmacological approaches. Progress in Neuro-Psychopharmacology and Biological Psychiatry, 27, 563579.

Fredriks, A.M., van Buuren, S., Wit, J.M., \& Verloove-Vanhorick, S.P. (2000). Body index measurements in 1996-7 compared with 1980. Archives of Disease in Childhood, 82, 107-112. 
Freedman, D.S., Khan, L.K., Dietz, W.H., Srinivasan, S.R., \& Berenson, G.S. (2001). Relationship of childhood obesity to coronary heart disease risk factors in adulthood: The Bogalusa Heart Study. Pediatrics, 108, 712-718.

Freijy, T., Mullan, B., \& Sharpe, L. (2014). Food-related attentional bias. Word versus pictorial stimuli and the importance of stimuli calorific value in the dot probe task. Appetite, 83, 202-208.

Gladwin, T.E., Figner, B., Crone, E.A., \& Wiers, R.W. (2011). Addiction, adolescence, and the integration of control and motivation. Developmental Cognitive Neuroscience, 1, 364-376. doi:10.1016/j.den.2011.06.008

Goldschmidt, A.B., Stein, R.I., Saelens, B.E., Theim, K.R., Epstein, L.H., \& Wilfley, D.E. (2011). Importance of early weight change in a pediatric weight management trial. Pediatrics, 12, 33-39.

Goossens, L., Braet, C., Verbeken, S., Decaluwe, V., \& Bosmans, G. (2011). Long-term outcome of pediatric eating pathology and predictors for the onset of loss of control over eating following weightloss treatment. International Journal of Eating Disorders, 44, 397-405, doi:10.1002/eat.20848

Graziano, P.A., Calkins, S.D., \& Keane, S.P. (2010). Toddler self-regulation skills predict risk for pediatric obesity. International Journal of Obesity, 34, 633-641, doi:10.1038/ijo.2009.288

Greenwald, A.G., Nosek, B.A., \& Banaji, M.R. (2003). Understanding and using the Implicit Association Test: I. An improved scoring algorithm. Journal of Personality and Social Psychology, 85, 197-216.

Greenwald, A.G., McGhee, D., \& Schwartz, J.L.K. (1998). Measuring individual differences in implicit cognition: The Implicit Association Test. Journal of Personality and Social Psychology, 74, 1464-1480.

Guerrieri, R., Nederkoorn, C., \& Jansen, A. (2008). The interaction between impulsivity and a varied food environment: Its influence on food intake and overweight. International Journal of Obesity, 32, 708-714.

Ho, M., Garnett, S.P., Baur, L.A., Burrows, T., Stewart, L., Neve, M., \& Collins, C. (2013), Impact of dietary and exercise interventions on weight change and metabolic outcomes in obese children and adolescents: A systematic review and meta-analysis of randomized trials. JAMA Pediatrics, 167, 759-768. doi:10.1001/jamapediatrics.2013.1453

Jelalian, E., Hart, C.N., Mehlenbeck, R.S., Lloyd-Richardson, E.E., Kaplan, J.D., Flynn-O’Brien, K.T., \& Wing, R.R. (2008). Predictors of attrition and weight loss in an adolescent weight control program. Obesity, 16, 1318-1323.

Kakoschke, N., Kemps, E., \& Tiggemannn, M. (2014). Attentional bias modification encourages healthy eating. Eating Behaviour, 15, 120-4. doi:10.1016/j.eatbeh.2013.11.001.

Karpinski, A., \& Steinman, R.B. (2006). The single category implicit association test as a measure of implicit social cognition. Journal of Personality and Social Psychology, 91, 16-32.

Kavanagh, D.J., Andrade, J., \& May, J. (2005). Imaginary relish and exquisite torture: The elaborated intrusion theory of desire. Psychological Review, 112, 446-467.

Kemps, E., Tiggemann, M., \& Hollitt, S. (2014). Biased attentional processing of food cues and modification in obese individuals. Health Psychology, 33, 1391-1401.

Kemps, E., \& Tiggemann, M. (2015). Approach bias for food cues in obese individuals. Psychology $\mathbb{E}$ Health, 30, 370-380.

Kemps, E., Tiggemann, M., \& Hollitt, S. (2016). Longevity of attentional bias modification effects for food cues in overweight and obese individuals. Psychology $\mathcal{E}$ Health, 31, 115-129.

Kemps, E., Tiggemann, M., Martin, R., \& Elliott, M. (2013). Implicit approach-avoidance associations for craved food cues. Journal of Experimental Psychology-Applied, 19, 30-38.

Kemps, E., Tiggemann, M., Orr, J., \& Grear, J. (2014). Attentional retraining can reduce chocolate consumption. Journal of Experimental Psychology Applied, 20, 94-102. doi:10.1037/xap0000005.

Koster, E.H.W., Fox, E., \& MacLeod, C. (2009). Introduction to the special section on cognitive bias modification in emotional disorders. Journal of Abnormal Psychology, 118, 1-4.

Kulendran, M., Vlaev, I., Sugden, C., King, D., Ashrafian, H., Gately, P., \& Darzi, A. (2014). Neuropsychological assessment as a predictor of weight loss in obese adolescents. International Journal of Obesity, 38, 507-512. 
Loeber, S., Grosshans, M., Korucuoglu, O., Vollmert, C., Vollstadt-Klein, S., Schneider, S., ... Kiefer, F. (2012). Impairment of inhibitory control in response to food-associated cues and attentional bias of obese participants and normal-weight controls. International Journal of Obesity, $36,1334-1339$.

Luttikhuis, H.O., Baur, L., Jansen, H., Shrewsbury, V.A., O’Malley, C., Stolk, R.P., \& Summerbell, C.D. (2009). Interventions for treating obesity in children, Cochrane Database of Systematic Reviews, 1 .

Mann, T., \& Ward, A, (2004). To eat or not to eat: Implications of the attentional myopia model for restrained eaters. Journal of Abnormal Psychology, 113, 90-98.

Moens, E., Braet, C., \& Van Winckel, M. (2010). An 8-year follow-up of treated obese children: Children's process and parental predictors of successful outcome. Behaviour Research and Therapy, 48, 626-633.

Murdaugh, D.L., Cox, J.E., Cook III, E.W., \& Weller, R.E. (2012). fMRI reactivity to high-calorie food pictures predicts short- and long-term outcome in a weight-loss program. Neurolmage, 59, 2709-2721.

Nederkoorn, C., Braet, C., Van Eijs, Y., Tanghe, A., \& Jansen, A. (2006). Why obese children cannot resist food: The role of impulsivity. Eating Behaviour, 7, 315-322. doi:org/10.1016/j.eatbeh.2005.11.005

Nederkoorn, C., Jansen, E., Mulkens, S., \& Jansen, A. (2007). Impulsivity predicts treatment outcome in obese children. Behaviour Research and Therapy, 45, 1071-1075.

Nijs, I.M.T., Franken, I.H.A., \& Muris, P. (2007). The modified Trait and State Food Cravings Questionnaires: Development and validation of a general index of food craving. Appetite, 49, $38-46$.

Pronk, T., van Deursen, D.S., Beraha, E.M., Larsen, H., \& Wiers, R.W. (2015). Validation of the Amsterdam Beverage Picture Set: A controlled picture set for cognitive bias measurement and modification paradigms. Alcoholism, Clinical EB Experimental Research, 39, 20472055

Raven, J.C. (1938). Progressive matrices: A perceptual test of intelligence. London: HK Lewis.

Rollins, B.Y., Dearing, K.K., \& Epstein, L.H. (2010). Delay discounting moderates the effect of food reinforcement on energy intake among non-obese women. Appetite, 55, 420-425.

Rosenbaum, M., Sy, M., Pavlovich, K., Leibel, R.L., \& Hirsch, J. (2008). Leptin reverses weight lossinduced changes in regional neural activity responses to visual food stimuli. Journal of Clinical Investigation, 118, 2583-2591. doi:10.1172/JCI35055.

Schoenmakers, T.M., de Bruin, M., Lux, I.F.M., Goertz, A.G., Van Kerkhof, D.H.A.T., \& Wiers, R.W. (2010). Clinical effectiveness of attentional bias modification training in abstinent alcoholic patients. Drug and Alcohol Dependence, 109, 30-36.

Seeyave, D.M., Coleman, S., Appugliese, D., Corwyn, R.F., Bradley, R.H., Davidson, N.S., Kaciroti, N., \& Lumeng, J. (2009). Ability to delay gratification at age 4 years and risk of overweight at age 11 years. Archives of Pediatrics $\mathcal{E}$ Adolescent Medicine, 163, 303-308.

Singh, A.S., Mulder, C., Twisk, J.W.R., van Mechelen, W., \& Chinapaw, M.J.M. (2008). Tracking of childhood overweight into adulthood: A systematic review of the literature. Obesity Reviews, 9, 474-488.

Skorka-Brown, J., Andrade, J., \& May, J. (2014). Playing 'Tetris' reduces the strength, frequency and vividness of naturally occurring cravings. Appetite, 76, 161-165.

Smith, E., Hay, P., Campbell, L., \& Trollor, J.N. (2011). A review of the association between obesity and cognitive function across the lifespan: Implications for novel approaches to prevention and treatment. Obesity Reviews, 12, 740-755.

Stoeckel, L.E., Weller, R.E., Cook III, E.W., Twieg, D.B., Knowlton, R.C., \& Cox, J.E. (2008). Widespread reward system activation in obese women in response to pictures of high-calorie foods. NeuroImage 41, 636-647.

Strack, F., \& Deutch, R. (2004). Reflective and impulsive determinants of social behavior. Personality and Social Psychology Review, 8, 220-247. 
Van Dillen, L.F., \& Andrade, J. (2016). Derailing the streetcar named desire. Cognitive distractions reduce individual differences in cravings and unhealthy snacking in response to palatable food. Appetite, 96, 102-110.

Verbeken, S., Braet, C., Bosmans, G., \& Goossens, L. (2014). Comparing decision-making in average and overweight children and adolescents. International Journal of Obesity, 38, 547-551.

Verbeken, S., Braet, C., Claus, L., Nederkoorn, C., \& Oosterlaan, J. (2009). Childhood obesity and impulsivity: An investigation with performance-based measures. Behaviour Change, 3, 153-167.

Verbeken, S., Braet, C., Goossens, L., \& van der Oord, S. (2013). Executive function training with game elements for obese children: A novel treatment to enhance self-regulatory abilities for weight-control. Behavior Research and Therapy, 51, 290-299. doi:10.1016/j.brat.2013.02.006.

Warschburger, P., Gmeiner, M., Morawietz, M., \& Rinck, M. (2017). Battle of plates: A pilot study of an approach-avoidance training for overweight children and adolescents. Public Health Nutrition, $21,426-434$.

Werthmann, J., Field, M., Roefs, A., Nederkoorn, C., \& Jansen, A. (2014). Attention bias for chocolate increases chocolate consumption - An attention bias modification study. Journal of Behavior Therapy and Experimental Psychiatry, 45, 136-43. doi:10.1016/j.jbtep.2013.09.009.

Weygandt, M., Mai, K., Dommes, E., Leupelt, V., Hackmack, K., Kahnt, T., ... Haynes, J.-D. (2013). The role of neural impulse control mechanisms for dietary success in obesity. Neuroimage, 83, 669-678.

Weygandt, M., Mai, K., Dommes, E., Ritter, K., Leupelt, V., Spranger, J., \& Haynes, J.-D. (2015). Impulse control in the dorsolateral prefrontal cortex counteracts post-diet weight regain in obesity. Neuroimage, 109, 318-327.

Whitaker, R.C., Wright, J.A., Pepe, M.S., Seidel, K.D., \& Dietz, WH. (1997). Predicting obesity in young adulthood from childhood and parental obesity. New England Journal of Medicine, 337, 869-873.

Wiers, R.W., Rinck, M., Kordts, R., Houben, K., \& Strack, F. (2010). Retraining automatic actiontendencies to approach alcohol in hazardous drinkers. Addiction, 105, 279-287.

Xanthakos, S.A., \& Inge, T.H. (2007). Extreme pediatric obesity: Weighing the health dangers. Journal of Pediatrics, 150, 3-5.

Yokum, S., Ng, J., \& Stice, E. (2011). Attentional bias to food images associated with elevated weight and future weight gain: An fMRI study. Obesity, 19, 1775-1783. 